\title{
Dislocations In Cubic Crystals Described By Discrete Models
}

\author{
Ana Carpio ${ }^{1}$, Luis L. Bonilla ${ }^{2}$, I. Plans ${ }^{2}$ \\ ${ }^{1}$ Departamento de Matemática Aplicada, Universidad Complutense \\ 28040 Madrid, Spain, ana_carpio@mat.ucm.es; \\ ${ }^{2}$ Departamento de Materiales, Universidad Carlos III, 28911 Leganés \\ Spain, bonilla@ing.uc3m.es, ignacio.plans@uc3m.es.
}

\begin{abstract}
Discrete equations of motion for dislocations in cubic crystals including dissipation and fluctuations are derived. These models have the standard linear anisotropic elasticity as their continuum limit and their main ingredients are the elastic stiffness constants of the material and a dimensionless periodic function that restores the translation invariance of the crystal and influences the dislocation size. Static and moving dislocations are identified with wave solutions of the model.
\end{abstract}

\section{Introduction}

Understanding mechanical, optical and electronic properties of materials requires a better understanding of the relation between defects and observed macroscopic behavior. The atomic scale can be resolved by ab initio or molecular dynamics simulations, which are very costly at the present time. Thus, it is interesting to have systematic models of defect motion in crystals that can be solved cheaply, are compatible with elasticity and yield useful information about the defect cores and their mobility.

In a previous paper, we have proposed a discrete model of dislocations and their motion in cubic crystals with a one atom basis [1]. Let us consider a simple cubic crystal. Firstly, we discretize space along the primitive vectors defining the unit cell of the crystal $\mathbf{x} \equiv(x, y, z)=(l, m, n) a$, in which $a$ is the length of the primitive cubic cell, and $l, m$ and $n$ are integer numbers. Secondly, we replace the gradient of the displacement vector $\tilde{u}_{i}(x, y, z, t)=a u_{i}(l, m, n ; t)\left(u_{i}(l, m, n ; t)\right.$ is a nondimensional vector $)$ in the strain energy density by an appropriate periodic function of the discrete gradient, $g\left(D_{j}^{+} u_{i}\right)$ : We shall define the discrete distortion tensor as

$$
\begin{aligned}
& w_{i}^{(j)}=g\left(D_{j}^{+} u_{i}\right), \\
& D_{1}^{ \pm} u_{i}(l, m, n ; t)= \pm\left[u_{i}(l \pm 1, m, n ; t)-u_{i}(l, m, n ; t)\right],
\end{aligned}
$$

etc., where $g(x)$ is a periodic function of period one satisfying $g(x) \sim x$ as $x \rightarrow 0$. The strain energy density for the discrete model is obtained by substituting the strain tensor in the usual strain energy density:

$$
\begin{aligned}
& W=\frac{1}{2} c_{i j k l} e_{i j} e_{k l}, \\
& c_{i j k l}=C_{12} \delta_{i j} \delta_{k l}+\frac{C_{11}-C_{12}}{2}\left(\delta_{i k} \delta_{j l}+\delta_{i l} \delta_{j k}\right)
\end{aligned}
$$




$$
\begin{aligned}
& +H\left(\frac{\delta_{i k} \delta_{j l}+\delta_{i l} \delta_{j k}}{2}-\delta_{1 i} \delta_{1 j} \delta_{1 k} \delta_{1 l}-\delta_{2 i} \delta_{2 j} \delta_{2 k} \delta_{2 l}-\delta_{3 i} \delta_{3 j} \delta_{3 k} \delta_{3 l}\right) \\
& H=2 C_{44}+C_{12}-C_{11} \\
& e_{i j}=\frac{1}{2}\left(w_{i}^{(j)}+w_{j}^{(i)}\right)=\frac{g\left(D_{j}^{+} u_{i}\right)+g\left(D_{i}^{+} u_{j}\right)}{2}
\end{aligned}
$$

(sum over repeated indices is assumed), where $c_{i j k l}$ are the elastic constants of the crystal. Summing over all lattice sites, we obtain the potential energy of the crystal:

$$
V\left(\left\{u_{i}\right\}\right)=a^{3} \sum_{l, m, n} W(l, m, n ; t)
$$

in which we have considered the strain energy deensity to be a function of the point $W(\mathbf{u})=W(l, m, n ; t),(l, m, n)=(x, y, z) / a$. Next, we find the equations of motion by the usual methods of classical mechanics. For conservative dynamics:

$$
\rho a^{4} \ddot{u}_{i}(l, m, n ; t)=-\frac{1}{a} \frac{\partial V\left(\left\{u_{k}\right\}\right)}{\partial u_{i}(l, m, n ; t)},
$$

or, equivalently [1],

$$
\rho a^{2} \ddot{u}_{i}=\sum_{j, k, l} D_{j}^{-}\left[c_{i j k l} g^{\prime}\left(D_{j}^{+} u_{i}\right) g\left(D_{l}^{+} u_{k}\right)\right]
$$

Here $\ddot{u}_{i} \equiv \partial^{2} u_{i} / \partial t^{2}$ and the displacement vector is dimensionless, so that both sides of Eq. (9) have units of force per unit area. Let us now restore dimensional units to Equation (9), so that $\tilde{u}_{i}(x, y, z)=a u_{i}(x / a, y / a, z / a)$, then let $a \rightarrow 0$, use Eq. (9) and that $g(x) \sim x$ as $x \rightarrow 0$. Then we obtain the usual Cauchy equations of linear elasticity:

$$
\rho \frac{\partial^{2} \tilde{u}_{i}}{\partial t^{2}}=\sum_{j, k, l} \frac{\partial}{\partial x_{j}}\left(c_{i j k l} \frac{\partial \tilde{u}_{k}}{\partial x_{l}}\right),
$$

provided the components of the distortion tensor are very small. Far from the core of a defect, the discrete gradient approaches the continuous one. Then, provided the slope $g^{\prime}(0)$ is one in the appropriate units, the spatially discrete equations of motion become those of the anisotropic elasticity. The periodic function $g(x)$ ensures that sliding a plane of atoms an integer number of times the lattice distance $a$ parallel to a primitive direction does not change the potential energy of the crystal.

Once the discrete model is specified, different dislocation configurations can be selected by requiring that their far field should adopt the well-known form of continuous elasticity $[1,2]$. Static dislocations move when the applied force surpasses a critical threshold. The magnitude of this threshold is controlled by the size of the regions where $g^{\prime}$ becomes negative: it decreases as their size increase. This information allows to fit $g$ for particular materials. A possible choice is:

$$
g(x)=\left\{\begin{array}{l}
x, \quad|x|<\frac{1}{2}-\alpha, \\
\frac{(1-2 \alpha)(1-2 x)}{4 \alpha}, \quad \frac{1}{2}-\alpha<x<\frac{1}{2}+\alpha,
\end{array}\right.
$$

which is periodically extended outside the interval $(\alpha-1 / 2, \alpha+1 / 2)$ for a given $\alpha \in$ $(0,1 / 2)$. To select $\alpha$, we calculate numerically the Peierls stress needed to move a given dislocation as a function of $\alpha$ and fit it to data from experiments or molecular dynamics calculations. 
In all cases, the procedure to obtain numerically the dislocation from the discrete model is the same. We first solve the stationary equations of elasticity with appropriate singular source terms to obtain the dimensional displacement vector $\tilde{\mathbf{u}}(x, y, z)=$ $\left(\tilde{u}_{1}(x, y, z), \tilde{u}_{2}(x, y, z), \tilde{u}_{3}(x, y, z)\right)$ of the static dislocation under zero applied stress. This displacement vector yields the far field of the corresponding dislocation for the discrete model, which is the nondimensional displacement vector:

$$
\mathbf{U}(l, m, n)=\frac{\tilde{\mathbf{u}}\left(\left(l+\delta_{1}\right) a,\left(m+\delta_{2}\right) a,\left(n+\delta_{3}\right) a\right)}{a} .
$$

Here $0 \leq \delta_{i}<1, i=1,2,3$, are chosen so that the singularity at $x=y=z=0$ does not coincide with a lattice point. For a sc crystal, it is often convenient to select the center of a unit cell, $\delta_{i}=1 / 2$. We use the nondimensional static displacement vector $\mathbf{U}(l, m, n)$ defined by (12) in the boundary and initial conditions for the discrete equations of motion.

Take for example, a pure screw dislocation along the $z$ axis with Burgers vector $\mathbf{b}=$ $(0,0, \mathrm{~b})$ has a displacement vector $\tilde{\mathbf{u}}=\left(0,0, \tilde{u}_{3}(x, y)\right)$ with $\tilde{u}_{3}(x, y)=\mathrm{b}(2 \pi)^{-1} \tan ^{-1}(y / x)$ [2]. The discrete equation for the $z$ component of the nondimensional displacement $u_{3}(l, m ; t)$ is:

$$
\rho a^{2} \ddot{u}_{3}=C_{44}\left\{D_{1}^{-}\left[g\left(D_{1}^{+} u_{3}\right) g^{\prime}\left(D_{1}^{+} u_{3}\right)\right]+D_{2}^{-}\left[g\left(D_{2}^{+} u_{3}\right) g^{\prime}\left(D_{2}^{+} u_{3}\right)\right]\right\} .
$$

To find the static solution of this equation corresponding to a screw dislocation, we could minimize an energy functional. However, it is more efficient to solve the following overdamped equation:

$$
\beta \dot{u}_{3}=C_{44}\left\{D_{1}^{-}\left[g\left(D_{1}^{+} u_{3}\right) g^{\prime}\left(D_{1}^{+} u_{3}\right)\right]+D_{2}^{-}\left[g\left(D_{2}^{+} u_{3}\right) g^{\prime}\left(D_{2}^{+} u_{3}\right)\right]\right\} .
$$

The stationary solutions of Eqs. (13) and (14) are the same, but the solutions of (14) relax rapidly to the stationary solutions if we choose appropriately the damping coefficient $\beta$. We solve Eq. (14) with initial condition $u_{3}(l, m ; 0)=U_{3}(l, m) \equiv \mathrm{b}(2 \pi a)^{-1} \tan ^{-1}[(m+$ $1 / 2) /(l+1 / 2)]$ (corresponding to $\delta_{i}=1 / 2$ ), and with boundary conditions $u_{3}(l, m ; t)=$ $U_{3}(l, m)+F m$ at the upper and lower boundaries of our lattice. At the lateral boundaries, we use zero-flux Neumann boundary conditions. Here $F$ is an applied dimensionless stress (the dimensional stress is $C_{44} F$ ). For small stresses, the solution of Eq. (14) relaxes to a static screw dislocation $u_{3}(l, m)$ with the desired far field. A static screw dislocation moves if the applied shear stress surpasses the static Peierls stress, $F_{c s}$. A moving dislocation continues doing so until the applied shear stress falls below a lower threshold $F_{c d}$ (dynamic Peierls stress); see [3] for a similar situation for edge dislocations. The numerical solution shows that moving a dislocation requires that we should have $g^{\prime}\left(D_{j}^{+} u_{3}\right)<0$ (with either $j=1$ or 2 ) at its core [3], which is harder to achieve as $\alpha$ decreases. A discusion of the changes in the size of the dislocation core and the Peierls stress due to $\alpha$ can be found in [1]. Using the same technique, stationary planar edge dislocations for an isotropic sc material have been constructed and a variety of dipole and loops of edge dislocations have been numerically found [1].

In this paper, we explain how to treat dissipative dynamics and the effect of fluctuations.

\section{Dissipative equations of motion}

Overdamped dynamics obtained by replacing the time differential of the displacement vector instead of the inertial term in the equation of motion (9) is not too realistic. 
Instead, we can add dissipation to the equations of motion by considering a quadratic dissipative function with cubic symmetry. The resulting equations are:

$$
\rho a^{2} \ddot{u}_{i}=\sum_{j} D_{j}^{-}\left[\left(\sigma_{i j}+\Sigma_{i j}\right) g^{\prime}\left(D_{j}^{+} u_{i}\right)\right]
$$

Here $\sigma_{i j}=c_{i j k l} e_{k l}=c_{i j k l} g\left(D_{l}^{+} u_{k}\right)$ is the elastic part of the stress tensor, and

$$
\begin{aligned}
\Sigma_{i j} & =\eta_{i j k l} \dot{e}_{k l} \\
\eta_{i j k l} & =\left(\zeta-\frac{2}{3} \eta\right) \delta_{i j} \delta_{k l}+\eta\left(\delta_{i k} \delta_{j l}+\delta_{i l} \delta_{j k}\right) \\
& +\gamma\left(\frac{\delta_{i k} \delta_{j l}+\delta_{i l} \delta_{j k}}{2}-\delta_{1 i} \delta_{1 j} \delta_{1 k} \delta_{1 l}-\delta_{2 i} \delta_{2 j} \delta_{2 k} \delta_{2 l}-\delta_{3 i} \delta_{3 j} \delta_{3 k} \delta_{3 l}\right)
\end{aligned}
$$

is the viscous part. In the cubic case, the viscosity tensor $\eta_{i k l m}$ is determined by the three scalar quantities $\zeta, \eta$ and $\gamma$. For isotropic sc crystals, $C_{11}=\lambda+2 \mu, C_{12}=\lambda, C_{44}=\mu$, $\gamma=0$. In the isotropic case and taking the continuum limit $a \rightarrow 0$, Eqs. (15) to (17) yield the viscous Navier's equations for isotropic elasticity [4]:

$$
\rho \frac{\partial^{2} \tilde{\mathbf{u}}}{\partial t^{2}}=\mu \Delta \tilde{\mathbf{u}}+(\lambda+\mu) \nabla(\nabla \cdot \tilde{\mathbf{u}})+\eta \Delta \frac{\partial \tilde{\mathbf{u}}}{\partial t}+\left(\zeta+\frac{\eta}{3}\right) \nabla\left(\nabla \cdot \frac{\partial \tilde{\mathbf{u}}}{\partial t}\right) .
$$

\section{Discrete model including dissipation and fluctuations}

To consider dissipation and fluctuations of the stress tensor and of the heat flux in the equation for the temperature we follow Onsager's ideas as used in Fluctuating Hydrodynamics [5] and obtain the equations of motion:

$$
\begin{aligned}
& \rho a^{2} \ddot{u}_{i}=\sum_{j} D_{j}^{-}\left[\left(\sigma_{i j}+\Sigma_{i j}+s_{i j}\right) g^{\prime}\left(D_{j}^{+} u_{i}\right)\right], \\
& \left\langle s_{i j}\right\rangle=0, \\
& \left\langle s_{i j}(l, m, n ; t) s_{a b}\left(l^{\prime}, m^{\prime}, n^{\prime} ; t^{\prime}\right)\right\rangle=k_{B} T \frac{\eta_{i j a b}+\eta_{a b i j}}{a^{3}} \delta_{l l^{\prime}} \delta_{m m^{\prime}} \delta_{n n^{\prime}} \delta\left(t-t^{\prime}\right),
\end{aligned}
$$

[with $\left.\sigma_{i j}=\sum_{k, l} c_{i j k l} g\left(D_{l}^{+} u_{k}\right)-\alpha_{i j}\left(T-T_{0}\right)\right]$ for the displacement vector, and

$$
\begin{aligned}
& \rho a c \dot{T}+a T \sum_{i, j} \alpha_{i j} g^{\prime}\left(D_{j}^{+} u_{i}\right) D_{j}^{+} \dot{u}_{i}=-\sum_{i} D_{i}^{-}\left(Q_{i}+\xi_{i}\right), \\
& \left\langle\xi_{i}\right\rangle=0, \\
& \left\langle\xi_{i}(l, m, n ; t) \xi_{j}\left(l^{\prime}, m^{\prime}, n^{\prime} ; t^{\prime}\right)\right\rangle=k_{B} T^{2} \frac{\kappa_{i j}+\kappa_{j i}}{a^{3}} \delta_{l l^{\prime}} \delta_{m m^{\prime}} \delta_{n n^{\prime}} \delta\left(t-t^{\prime}\right),
\end{aligned}
$$

for the local temperature $T(l, m, n)$. In Eq. (21), $c$ is the specific heat of the solid, and the heat flux $Q_{i}$ is related to the discrete gradient of the temperature by the constitutive relation $Q_{i}=-\sum_{j} \kappa_{i j} D_{j}^{+} T / a$, where $\kappa_{i j}$ is the thermal conductivity tensor. $k_{B}$ is the Boltzmann constant and the temperature is measured in Kelvin. The correlations of the fluctuating stress tensor and the fluctuating heat flux contain the viscosity tensor and the thermal conductivity tensor, respectively. In crystals with cubic symmetry, the elastic constants and the viscosity tensor are given by Eqs. (4) and (17), respectively. The thermal conductivity and thermal expansion tensors are isotropic, $\kappa_{i j}=\kappa \delta_{i j}, \alpha_{i j}=\alpha \delta_{i j}$. Note that the correlations of $s_{i j}$ in $(20)$ and of $\xi_{i}$ in (22) are proportional to $1 / a^{3}$, which becomes $\delta\left(\mathbf{x}-\mathbf{x}^{\prime}\right)$ in the continuum limit as $a \rightarrow 0$. 


\section{Extensions to fcc and bcc crystals}

Let us explain how to extend our discrete models of dislocations to fcc or bcc crystal symmetry, assuming that we have one atom per lattice site. For fcc or bcc crystals, the primitive vectors of the unit cell are not orthogonal. To find a discrete model for these crystals, we should start by writing the strain energy density in a non-orthogonal vector basis, $a_{1}, a_{2}, a_{3}$, instead of the usual orthonormal vector basis $e_{1}, e_{2}, e_{3}$ determined by the cube sides. Let $x_{i}$ denote coordinates in the basis $e_{i}$, and let $x_{i}^{\prime}$ denote coordinates in the basis $a_{i}$. Notice that the $x_{i}$ have dimensions of length while the $x_{i}^{\prime}$ are dimensionless. The matrix $\mathcal{T}=\left(a_{1}, a_{2}, a_{3}\right)$ whose columns are the coordinates of the new basis vectors in terms of the old orthonormal basis can be used to change coordinates as follows:

$$
x_{i}^{\prime}=\mathcal{T}_{i j}^{-1} x_{j}, x_{i}=\mathcal{T}_{i j} x_{j}^{\prime}
$$

Similarly, the displacement vectors in both basis are related by

$$
u_{i}^{\prime}=\mathcal{T}_{i j}^{-1} \tilde{u}_{j}, \tilde{u}_{i}=\mathcal{T}_{i j} u_{j}^{\prime}
$$

and partial derivatives obey

$$
\frac{\partial}{\partial x_{i}^{\prime}}=\mathcal{T}_{j i} \frac{\partial}{\partial x_{j}}, \frac{\partial}{\partial x_{i}}=\mathcal{T}_{j i}^{-1} \frac{\partial}{\partial x_{j}^{\prime}} .
$$

Note that $u_{i}^{\prime}$ and $x_{i}^{\prime}$ are nondimensional while $\tilde{u}_{i}$ and $x_{i}$ have dimensions of length. By using these equations, the strain energy density $W=(1 / 2) c_{i k l m} e_{i k} e_{l m}$ can be written as

$$
W=\frac{1}{2} c_{i j l m} \frac{\partial \tilde{u}_{i}}{\partial x_{j}} \frac{\partial \tilde{u}_{l}}{\partial x_{m}}=\frac{1}{2} c_{r s p q}^{\prime} \frac{\partial u_{r}^{\prime}}{\partial x_{s}^{\prime}} \frac{\partial u_{p}^{\prime}}{\partial x_{q}^{\prime}},
$$

where the new elastic constants are:

$$
c_{\text {rspq }}^{\prime}=c_{i j l m} \mathcal{T}_{i r} \mathcal{T}_{s j}^{-1} \mathcal{T}_{l p} \mathcal{T}_{q m}^{-1}
$$

Notice that the elastic constants have the same dimensions in both the orthogonal and the non-orthogonal basis. To obtain a discrete model, we shall consider that the dimensionless displacement vector $u_{i}^{\prime}$ depends on dimensionless coordinates $x_{i}^{\prime}$ that are integer numbers $u_{i}^{\prime}=u_{i}^{\prime}(l, m, n ; t)$. As in the simple cubic case, we replace the distortion tensor (gradient of the displacement vector in the non-orthogonal basis) by a periodic function of the corresponding forward difference, $w_{i}^{(j)}=g\left(D_{j}^{+} u_{i}^{\prime}\right)$. As in Eq. (11), $g$ is a periodic function with $g^{\prime}(0)=1$ and period 1 . The discretized strain energy density is

$$
W(l, m, n ; t)=\frac{1}{2} c_{r s p q}^{\prime} g\left(D_{s}^{+} u_{r}^{\prime}\right) g\left(D_{q}^{+} u_{p}^{\prime}\right) .
$$

The elastic constants $c_{r s p q}^{\prime}$ in (27) can be calculated in terms of the Voigt stiffness constants for a cubic crystal, $C_{11}, C_{44}$ and $C_{12}$, which determine the tensor of elastic constants (4). The elastic energy can be obtained from Eq. (28) for $W$ by means of Eqs. (7). Then the conservative equations of motion (8) are

$$
\rho a^{3} \frac{\partial^{2} u_{i}^{\prime}}{\partial t^{2}}=-\mathcal{T}_{i q}^{-1} \mathcal{T}_{p q}^{-1} \frac{\partial V}{\partial u_{p}^{\prime}},
$$


which, together with Eqs. (7) and (28), yield

$$
\rho \frac{\partial^{2} u_{i}^{\prime}}{\partial t^{2}}=\mathcal{T}_{i q}^{-1} \mathcal{T}_{p q}^{-1} D_{j}^{-}\left[g^{\prime}\left(D_{j}^{+} u_{p}^{\prime}\right) c_{p j r s}^{\prime} g\left(D_{s}^{+} u_{r}^{\prime}\right)\right]
$$

This equation becomes (9) for orthogonal coordinates, $\mathcal{T}_{i q}^{-1}=\delta_{i q} / a$.

To add dissipation and fluctuations to these equations, we need to replace $c_{p j r s}^{\prime} g\left(D_{s}^{+} u_{r}^{\prime}\right)$ by $c_{p j r s}^{\prime} g\left(D_{s}^{+} u_{r}^{\prime}\right)-\alpha_{p j}^{\prime}\left(T-T_{0}\right)+\eta_{p j r s}^{\prime} g^{\prime}\left(D_{s}^{+} u_{r}^{\prime}\right) D_{s}^{+} \dot{u}_{r}^{\prime}+s_{p j}^{\prime}$, in which $\eta_{p j r s}^{\prime}$ is related to the viscosity tensor (17) in the same way as $c_{p j r s}^{\prime}$ is related to $c_{i j l m}$ by (27). The random stress tensor $s_{p j}^{\prime}$ has zero mean and correlation given by (20) with the modified viscosity tensor $\eta_{i j a b}^{\prime}$ instead of the viscosity tensor (17). The heat conduction equations are

$$
\begin{aligned}
& \rho c \frac{\partial T}{\partial t}+T \alpha_{i j}^{\prime} g^{\prime}\left(D_{j}^{+} u_{i}^{\prime}\right) D_{j}^{+} \frac{\partial u_{i}^{\prime}}{\partial t}=D_{i}^{-}\left(\kappa_{i j}^{\prime} D_{j}^{+} T+\frac{\xi_{i}^{\prime}}{a}\right) \\
& \left\langle\xi_{i}^{\prime}\right\rangle=0 \\
& \left\langle\xi_{i}^{\prime}(l, m, n ; t) \xi_{j}^{\prime}\left(l^{\prime}, m^{\prime}, n^{\prime} ; t^{\prime}\right)\right\rangle=k_{B} T^{2} \frac{\kappa_{i j}^{\prime}+\kappa_{j i}^{\prime}}{a} \delta_{l l^{\prime}} \delta_{m m^{\prime}} \delta_{n n^{\prime}} \delta\left(t-t^{\prime}\right), \\
& \kappa_{p q}^{\prime}=\mathcal{T}_{p i}^{-1} \mathcal{T}_{q j}^{-1} \kappa_{i j}, \quad \alpha_{p q}^{\prime}=\frac{1}{2}\left(\mathcal{T}_{i p} \mathcal{T}_{q j}^{-1}+\mathcal{T}_{j p} \mathcal{T}_{q i}^{-1}\right) \alpha_{i j} .
\end{aligned}
$$

Note that the both the original and the modified tensors $\alpha_{i j}$ and $\kappa_{i j}$ are symmetric.

Once we have derived the equations of motion, stationary dislocations can be calculated by first finding the corresponding solution to the equations of anisotropic elasticity and using it to set up initial and boundary conditions for overdamped equations of motion. For fcc and bcc crystals, screw and edge dislocations have been constructed in [1].

\section{Conclusions}

We have proposed discrete models describing defects in crystal structures whose continuum limit is the standard linear anisotropic elasticity, including fluctuations according to the fluctuation-dissipation theorem. The main ingredients entering the models are the elastic stiffness constants of the material and a dimensionless periodic function that restores the translation invariance of the crystal (and together with the elastic constants determines the dislocation size). For simple cubic crystals, their equations of motion with conservative or damped dynamics are derived. For fcc and bcc metals, the primitive vectors along which the crystal is translationally invariant are not orthogonal. Similar discrete models and equations of motion are found by writing the strain energy density and the equations of motion in non-orthogonal coordinates. These models to investigate dislocation motion and interaction in simple geometries.

\section{Acknowledgements}

This work has been supported by the Spanish MEC Grant MAT2005-05730-C02.

\section{References}

[1] Carpio, A., Bonilla, L.L., 2005, Discrete models for dislocations and their motion in cubic crystals, Phys. Rev. B 71, 134105. 
[2] Hirth, J.P., Lothe, J., 1982. Theory of Dislocations. John Wiley \& Sons, New York.

[3] Carpio, A., Bonilla, L.L., 2003. Edge dislocations in crystal structures considered as traveling waves of discrete models. Phys. Rev. Lett. 90, 135502.

[4] Landau, L.D., Lifshitz, E.M., 1986. Theory of elasticity. Pergamon Press, London.

[5] Landau, L.D., Lifshitz, E.M., 1959. Fluid Mechanics, Pergamon Press, London. 